\title{
Impact of reflection on the fluence rate distribution in a UV reactor with various inner walls as measured using a micro-fluorescent silica detector
}

\author{
Mengkai Li ${ }^{a}$, Zhimin Qiang ${ }^{a, *}$, James R. Bolton ${ }^{b}$, Weiwei Ben ${ }^{a}$ \\ a State Key Laboratory of Environmental Aquatic Chemistry, Research Center for Eco-Environmental Sciences, Chinese Academy of Sciences, \\ 18 Shuang-qing Road, Hai-dian District, Beijing 100085, China \\ ${ }^{\mathrm{b}}$ Department of Civil and Environmental Engineering, University of Alberta, Edmonton, AB T6G 2W2, Canada
}

\section{A R T I C L E I N F O}

Article history:

Received 23 January 2012

Received in revised form

30 March 2012

Accepted 2 April 2012

Available online 10 April 2012

Keywords:

UV reactor

Inner-wall reflection

Fluence rate distribution

In-situ measurement

Micro-fluorescent silica detector

\begin{abstract}
A B S T R A C T
An assessment of the impact of ultraviolet (UV) reflection from inner walls is important for the accuracy of model predictions of fluence rate (FR) distribution and for the improvement of reactor efficiency. In this study, the FR distribution in an annular UV reactor with inner walls of various reflectances was measured in-situ by using a $360^{\circ}$ response microfluorescent silica detector. The tests were performed in water with various transmittances ranging from $65 \%$ to $99 \%$ and with inner reactor walls composed of quartz/ aluminum foil, quartz/stainless steel, or quartz/black cloth, whose reflection coefficients were determined to be $80.5 \%, 26.1 \%$ and $11.1 \%$, respectively. The results demonstrate that an inner wall with a high reflection coefficient can lead to a marked increase in the weighted average FRs, thus greatly improving the reactor efficiency. Furthermore, the presently used FR distribution models could have an error of up to $35 \%$ for commonly used stainless steel walls as a result of the influence of inner-wall reflection. Finally, it was found that the uniformity of the FR distribution is strongly dependent on the diffuse reflection property of the inner wall, which could lead to a better fluence delivery distribution in the UV reactor. This work has potential application to increase the accuracy of model predictions as well as optimize the design of high-efficiency UV reactors.
\end{abstract}

(c) 2012 Elsevier Ltd. All rights reserved.

\section{Introduction}

In recent years, ultraviolet (UV) technology has been used increasingly around the world for disinfection and photochemical oxidation of water and wastewater. One of the challenging problems regarding the application of UV technology is the lack of fast and cost-effective direct confirmation of the delivered fluence (or UV dose) in a UV reactor (Qualls and Johnson, 1983; Blatchley et al., 2008; Mamane-Gravetz et al., 2005; Bohrerova et al., 2005). To calculate the UV dose accurately with numerical models, the spatial fluence rate (FR) distribution in a UV reactor is a prerequisite. In addition, a combination of an FR distribution model with a computational fluid dynamics model allows the optimization of the configuration of a UV reactor to achieve higher efficiencies (Sozzi and Taghipour, 2006; Elyasi and Taghipour, 2010a; Chen et al., 2011).

Some numerical models have been developed for the determination of the FR distribution in UV reactors including the point source summation (PSS) (Jacob and Dranoff, 1970), line source integration (LSI) (Blatchley, 1997) and extense source with volumetric emission (ESVE) (Irazoqui et al., 1973,

\footnotetext{
* Corresponding author. Tel.: +8610 62849632; fax: +861062923541.

E-mail address: qiangz@rcees.ac.cn (Z. Qiang). 
2000) models. Bolton (2000) developed a model that considered some key factors, such as reflection and refraction of UV at the quartz sleeve and the absorbance of water. This model, now commercially available as UVCalc ${ }^{\circledR}$ (Bolton Photosciences Inc., Edmonton, AB, Canada), incorporates the multiple segment source summation (MSSS) approximation. However, none of the above-mentioned models takes account of inner-wall reflection. In addition, complex optical behaviors exist in UV reactors, and some crucial model parameters may not be determined accurately. Hence, direct measurements of FR distributions in a UV reactor provide a desirable and necessary tool for validation of numerical models.

Many researchers have attempted to verify the FR models experimentally (Jin et al., 2005; Rahn et al., 2006; Quan et al., 2005; Imoberdorf et al., 2008; Liu et al., 2004; Elyasi and Taghipour, 2010a,b). For example, to better represent the FR exposed to microorganisms in a UV reactor that receive photons from all angles, spherical actinometry has been employed by using spherical quartz vessels containing the iodide/iodate actinometer as a detector (Jin et al., 2005; Rahn et al., 2006; Liu et al., 2004). However, the positions of the spheres could not be controlled accurately, and the large diameter $(1.0 \mathrm{~cm})$ decreased the measurement accuracy near the lamp. Furthermore, Elyasi and Taghipour (2010a,b) used a radiometer-type detector to measure the $\mathrm{FR}$ distribution. This detector had a small active surface $(0.5 \mathrm{~mm} \times 0.5 \mathrm{~mm})$ that approximates a point detector. The lamp and the detector were respectively fixed on two linear-stage rails, and so could move parallel or perpendicular to the lamp axis, thus increasing the position precision.

Nevertheless, to date there has been very limited research on the impact of reflection by UV beams at the inner reactor wall, either in model calculations or in experimental measurements. Sommer et al. (1996) experimentally investigated the reflection effect from the inner wall. A distinct decrease in the reduction equivalent UV dose (RED) was found after eliminating the reflection by black lacquering the inner surface of a UV reactor. Some other researchers (Imoberdorf et al., 2008; Chen et al., 2011) investigated the impact of inner-wall reflection on the FR distribution through model calculations. In fact, the FR values are lower in the near-wall region than those in other regions of a UV reactor, which can significantly impact UV dose delivery. As a result, an accurate measurement of the impact of inner-wall reflection on the FR distribution would be useful, not only for developing and validating FR models, but also for optimizing the design of UV reactors to achieve higher efficiencies.

In our previous research (Li et al., 2011) a micro-fluorescent silica detector (MFSD) was developed for in-situ measurement of FR distribution in a UV reactor. The key features of this $360^{\circ}$ response detector, such as high stability, fast response, waterresistance and small volume, are particularly suitable for measuring the impact of reflection from inner walls on the FR distribution. In addition, a reference MFSD could be inserted into the gap between the lamp and the quartz sleeve and thus monitor any fluctuation of the lamp output, which significantly increased the measurement precision.

This work aimed to clarify, by use of the MFSD, the impact of inner-wall reflection on the FR distribution in a UV reactor. Three different inner-wall materials were used, namely, quartz/aluminum foil (QAF), quartz/stainless steel (QSS), and quartz/black cloth (QBC). Moreover, the detailed effect of water transmittance (UVT) (ranging from 65\% to 99\%) on the FR distribution was examined. This study has a potential application to increase the model precision and to optimize the design of high-efficiency UV reactors.

\section{Materials and methods}

Fig. 1 shows a schematic diagram of the experimental set-up (i.e., the entire test system and the UV reactor) for the measurement of FR distributions under the impact of reflective inner walls. A cylindrical quartz UV reactor (>98\% quartz transmittance measured at $254 \mathrm{~nm}$ and 1-cm path length with

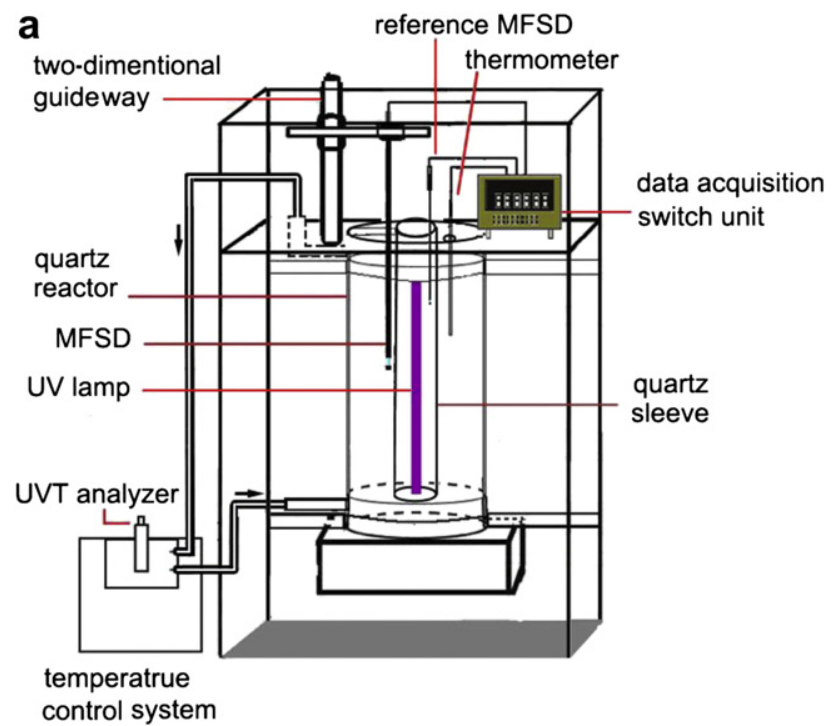

b

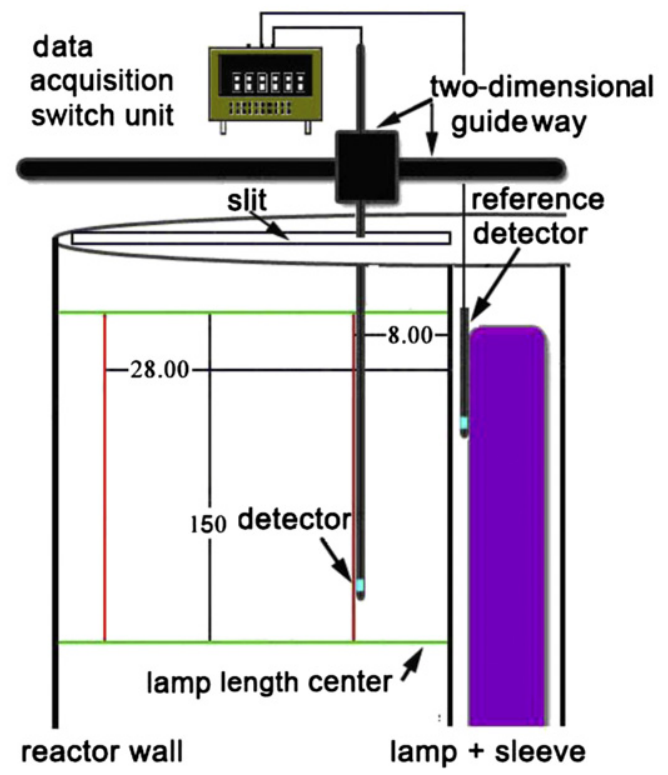

Fig. 1 - Schematic diagram of the experimental set-up for measurement of the fluence rate distribution under the impact of reflective inner walls: (a) the entire test system; (b) the UV reactor. 
the reflection contribution taken out) was employed with an inner diameter of $95 \mathrm{~mm}$ and an inner height of $400 \mathrm{~mm}$. Quartz was chosen as the reactor wall, which replaced the commonly used stainless steel. This permitted the wrapping of various reflective materials around the outside surface of the reactor and still maintained the same reactor operating status. A low-pressure mercury UV lamp (GL Type, Xiashi Wanhua Co., China) was housed in the center of the UV reactor, around which a quartz sleeve (23 $\mathrm{mm}$ o.d.) was placed. The lamp parameters were: arc length $=297 \mathrm{~mm}$; total output power $=16 \mathrm{~W}$; UVC efficiency at $254 \mathrm{~nm}=26 \%$ (calculated from the FR measured by the MFSD $1.0 \mathrm{~m}$ away from the lamp).

A cylindrical MFSD (1.0 mm long and $0.3 \mathrm{~mm}$ o.d.) was used as the UV detector, where its axial axis was always oriented parallel to the lamp. This small detector can capture photons from nearly all directions and hence was particularly suitable for the measurement of the influence of reflection from the inner wall. The UV-excited fluorescence from the MFSD was received by a BPW $34 \mathrm{~B}$ silicon PIN photodiode, transmitted by an optical fiber, and amplified and displayed by a multimeter. The MFSD calibration process was the same as that described in a previous paper (Li et al., 2011). The detector was fixed onto a two-dimensional guide way, which could move parallel or perpendicular to the axis of the lamp, and then inserted into the reactor chamber through a slit open in the upper cover of the reactor.

It has been determined in our laboratory (unpublished data) that the MFSD has an angular response in directions away from the detector axis. A detailed analysis of this effect indicates that the observed $F R$ readings should be increased by $8 \%$ to account for this angular response. Consequently, all FR measurements in this paper represent the corrected values.

A second MFSD, used as the reference detector, was inserted into the gap between the lamp and the sleeve to monitor any lamp output fluctuations. The displacement precisions of the guide way parallel and perpendicular to the axis of the lamp were \pm 1 and $\pm 0.01 \mathrm{~mm}$, respectively. The water temperature was controlled by a recirculator with a precision of $\pm 0.5^{\circ} \mathrm{C}$.

To assess the influence of various inner walls, aluminum foil, stainless steel, or black cloth (made of cotton) was placed individually around the outside surface of the quartz UV reactor (i.e., QAF, QSS, and QBC) as the reflective material. For the stainless steel, a stainless steel tube (Type 304, $100 \mathrm{~mm}$ i.d. $\times 1 \mathrm{~mm}$ thickness), whose inside surface had been mechanically polished, was cut into two equal semi-circular parts. During the tests, the two parts were combined together around the reactor. In addition, it was determined that the black cloth was totally opaque to $254 \mathrm{~nm} \mathrm{UV.}$

Potassium acid phthalate solutions (with UVTs of $99 \%, 95 \%$, $92.5 \%, 90 \%, 87.5 \%, 82.5 \%, 75 \%$ and $65 \%$, respectively, as determined with a spectrophotometer (DR5000, Hach Co., USA)) were used as the media. For each medium, the detector was placed parallel to the UV lamp at radial distances of 8.00 and $28.00 \mathrm{~mm}$ away from the quartz sleeve. At a fixed radial distance, the detector was moved vertically to measure the FR values at pre-selected test points in the UV reactor. The detector stayed at each test point for $1 \mathrm{~min}$ when a data acquisition switch unit (34971A, Agilent Co., USA) collected thirty readings. Then the last ten readings were recorded and averaged. The final result for each test point was divided by the corresponding reading of the reference detector to correct any potential error induced by the lamp output fluctuation.

The commercial UVCalc ${ }^{\circledR}$ MSSS FR model was chosen to compare with the experimental measurements, since it showed the best agreement with experimental data among five tested models (Liu et al., 2004).

The spectral reflection coefficients were measured in the wavelength range from 210 to $280 \mathrm{~nm}$ for each reflective material by using a spectrophotometer (Model lambda 950, PerkinElmer, USA) equipped with an integrating ball accessory. The diffuse reflection property was measured by use of a vacuum UV analytical spectrophotometer (VUVAS 2000, McPherson Inc., USA), as illustrated in Fig. S1. A magnesiumfluoride windowed deuterium lamp, coupled with a light gathering reflective condenser and an optimized monochromator, was used as the light source emitting collimated UV beams at $254 \mathrm{~nm}$. Two chopped UV beams were used in the test (one incident on the sample plate and the other used as a reference signal). The sample plate and the detector could both rotate independently to receive signals for various incident angles (i.e., $20^{\circ}, 40^{\circ}$, and $60^{\circ}$ in this work) and various acceptance angles to determine the diffuse reflection properties of the test material. In the above measurements, each reflective material was placed behind a quartz sheet $(2 \mathrm{~mm}$ thickness) to simulate the experimental conditions of the quartz UV reactor with various inner walls (i.e., QAF, QSS, and $\mathrm{QBC})$.

\section{Results and discussion}

\subsection{Reflection coefficient spectra of various inner walls}

Fig. 2 shows the reflection coefficient spectra of the three innerwall materials. At $254 \mathrm{~nm}$, the reflection coefficients of the QAF, QSS and QBC layers were determined to be $80.5 \%, 26.1 \%$ and $11.1 \%$, respectively. Since the test materials were covered with a quartz sheet, these results may slightly differ from those reported in the literature where no quartz sheet was used during the test. It is noted that a quartz sheet can absorb $(<2 \%)$ and reflect UV ( $8 \%$ for beams normal to the surface and higher for beams at a larger angle) (Bolton, 2010), and most of the UV reflectance of QBC can be attributed to the quartz. In the UV reactor, because the inner quartz surface is in contact with water, reflection from that surface will be much less (reflection coefficient 0.0025 for a water/quartz surface versus 0.0421 for an air/quartz surface). Hence, in the water only about $4.5 \%$ of incident UV light is reflected from the quartz itself.

\subsection{Fluence rate distribution measurements}

Figs. 3 and 4 show the FR distributions parallel to the axis of the lamp at radial distances of 8 and $28 \mathrm{~mm}$ from the sleeve, respectively. The addition of a reflective layer demonstrates that the FR at each point increases as the reflection coefficient of the inner-wall material increases. In addition, the FR increment depended not only on the UVT of water, but also on the reflection coefficient of the test material. 


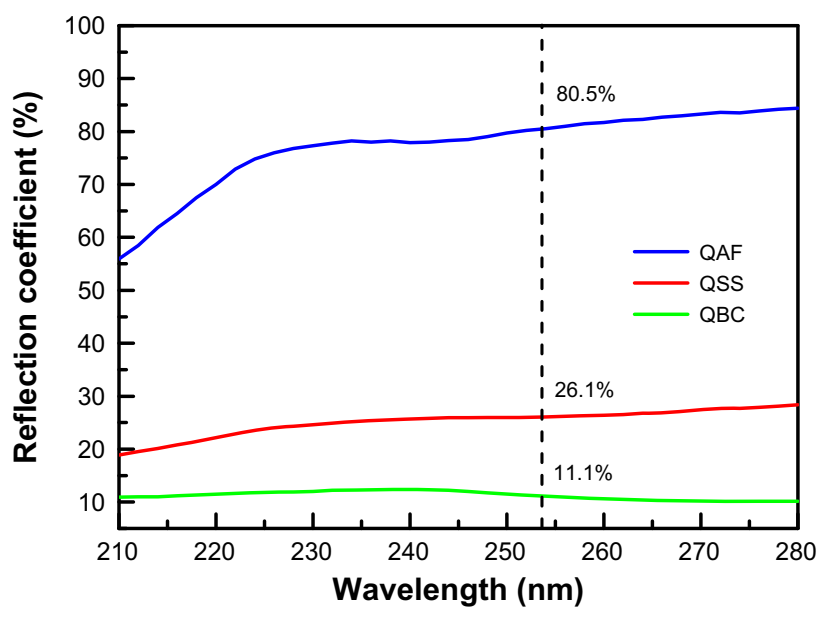

Fig. 2 - Reflection coefficient spectra of various inner-wall materials.

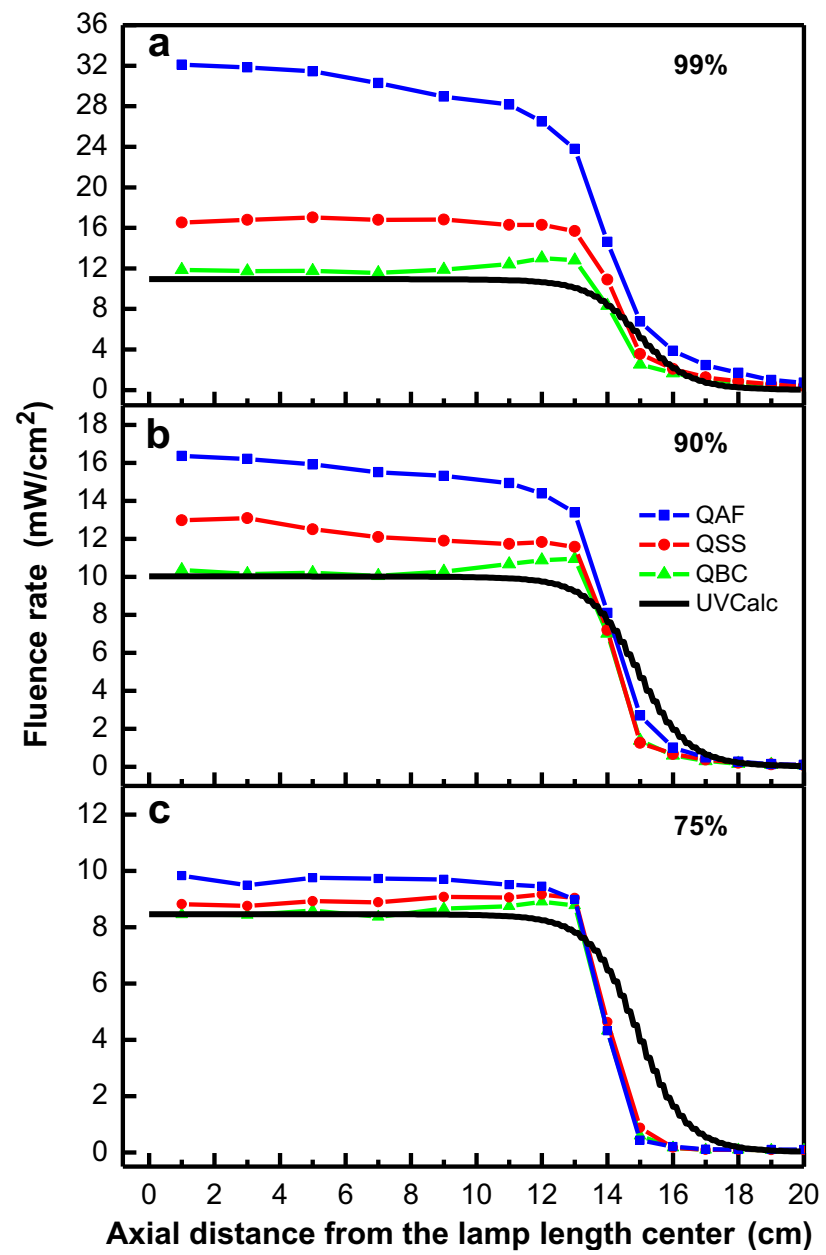

Fig. 3 - Fluence rate distribution parallel to lamp with various inner-wall materials in water with different UVTs at an $8 \mathrm{~mm}$ radial distance from the sleeve: (a) $99 \%$, (b) $90 \%$, and (c) $75 \%$.

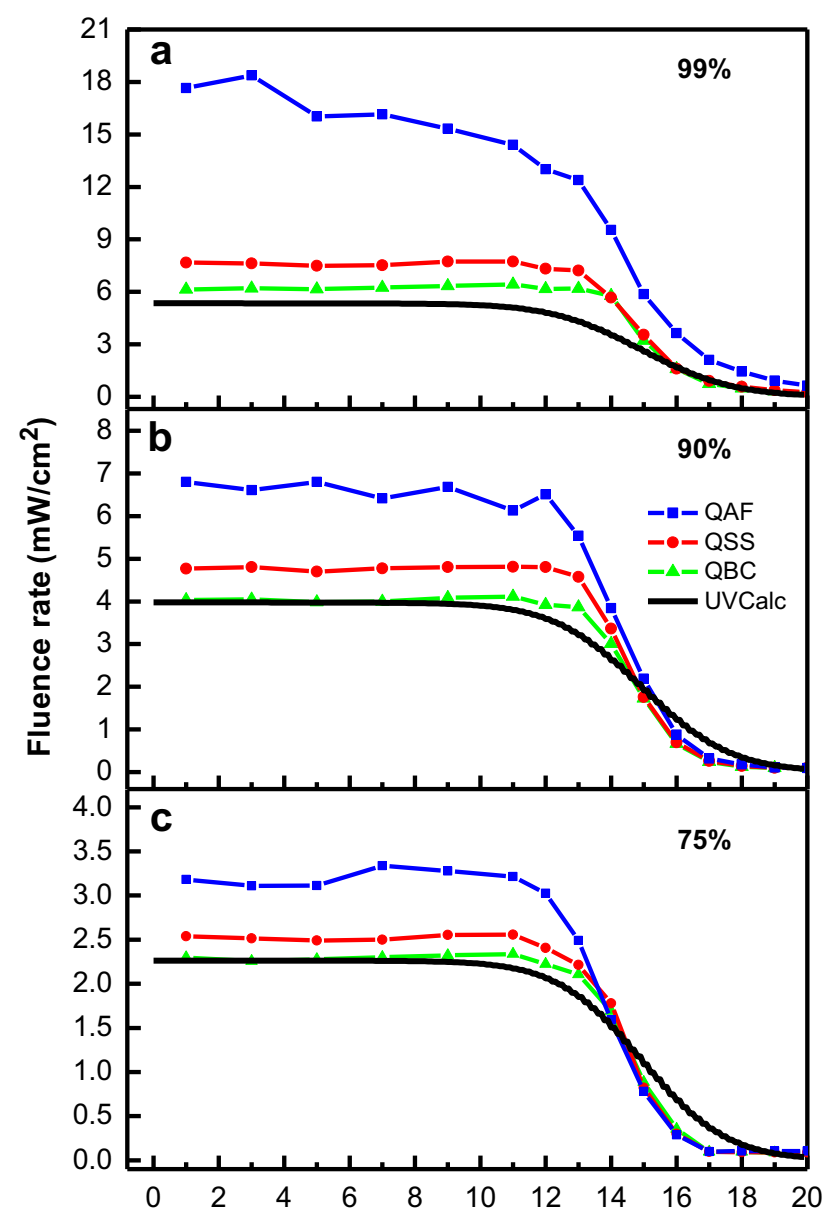

Axial distance from the lamp length center $(\mathrm{cm})$

Fig. 4 - Fluence rate distribution parallel to lamp with various inner-wall materials in water with different UVTs at a $28 \mathrm{~mm}$ radial distance from the sleeve: (a) $99 \%$, (b) $90 \%$, and (c) $75 \%$.

The UV beams impinging on the inner wall should be partially reflected depending on the reflection coefficient of the inner wall. The reflected UV beams re-enter the water and add to the test point readings. The extent of reflection is also affected by the UVT of water. In general, as expected, the FR increases more by using a reflective inner wall in the case of higher UVT water.

The results from the UVCalc ${ }^{\circledR}$ model are also presented (solid black curves), which agreed reasonably well with the results of QBC case, particularly in the principal part of the lamp length (i.e., 0-13 cm vertically from the lamp center). Since the QBC could also reflect some UV (reflection coefficient $=11.1 \%$ ), the measurements for higher UVT water (e.g., 99\%) were slightly higher than those calculated by $\mathrm{UVCalc}^{\circledR}$, which does not consider any influence of inner-wall reflection. For the QSS and QAF walls, the FR curves were markedly above the UVCalc $^{\circledR}$ curves because these two materials have a much higher reflection coefficients than does the QBC wall.

As illustrated in Figs. 3a and 4a (UVT $=99 \%$ ), when the detector moved parallel to the axis of the lamp from 0 to $11 \mathrm{~cm}$ 
relative to the lamp center, the $\mathrm{FR}$ with the $\mathrm{QBC}$ wall, as expected, remained almost the same, while the FR with the QAF wall increased toward the lamp center. This arises from the fact that in a higher UVT (e.g., 99\%) water with a higher reflecting material (e.g., QAF), the test points approaching the lamp center can receive more reflected UV beams from the inner wall. However, as the water UVT decreased, the increment in FR became smaller with the increasing reflection coefficient of the reflective material. For example, at a UVT of $75 \%$, the impacts of the three materials were minimal (see Figs. $3 \mathrm{c}$ and $4 \mathrm{c}$ ). It is also noted that unusual FR increases were found at $11-13 \mathrm{~cm}$ with the QBC in Fig. 3. This probably arises from the reflection of the top water level (ca. $17 \mathrm{~cm}$ from the lamp center) in the UV reactor. The UV beams in the water would be reflected by the water/air interface obeying the Fresnel's law, thus somewhat increasing the FR below the water level. Smaller effects were found far from the water level (i.e., 0-11 cm). However, reflection by the inner wall predominated when a higher reflecting material (e.g., QAF or QSS) was used, which overwhelmed the influence from the water/air interface (Fig. 3). Fig. 4 shows a similar trend, but at a reduced level, because of the lower FR values that were measured at a larger radial distance $(28 \mathrm{~mm})$ from the sleeve.

Fig. 5 shows the FR distribution as a function of the radial distance from the sleeve in the central cross-section of the UV reactor. The UVT of water varied from $75 \%$ to $99 \%$. These results indicate that the FR distribution with a higher reflecting material (e.g., QAF in Fig. 5a) was impacted more strongly by the UVT of water than that with a lower reflecting material (e.g., QBC in Fig. 5c), especially in the near-sleeve region (e.g., at $3 \mathrm{~mm}$ distance from the sleeve). This is reasonable, since the UV beams would be reflected more strongly by a higher reflecting material, and thus were more obviously influenced by the UVT of water. Moreover, a high UVT water tended to induce multiple-time reflections by the inner wall, which would also increase the FR values. In addition, in the nearsleeve region, the FR with the $\mathrm{QBC}$ wall arose principally from UV beams directly emitted from the lamp, while that with the QAF or QSS wall had a significant component of reflected UV beams. Therefore, the UVT impact in this region was more obvious for a higher reflecting material (e.g., QAF). It is also noted that all the FR distribution curves showed good regularity and repeatability. In the past, it was very difficult to measure the FRs in the near-sleeve region. However, this problem is well resolved in this work because of the applicative properties of the MFSD, the high precision in positioning the detector, and the correction of lamp output fluctuation by using a reference MFSD.

\subsection{Weighted average fluence rates}

The ideal fluence (or UV dose) in a UV reactor can be estimated generally by multiplying the volumetrically averaged FR by the hydraulic retention time. It is recognized that the fluence in real UV reactors is actually a distribution that will be narrow or broad depending on whether hydraulic mixing is good or poor compared to the perfect mixing case (i.e., perfect radial mixing and zero longitudinal mixing). Nevertheless, the ideal fluence should change in a manner very similar to the distribution of the real fluence in a UV reactor. Likewise, the

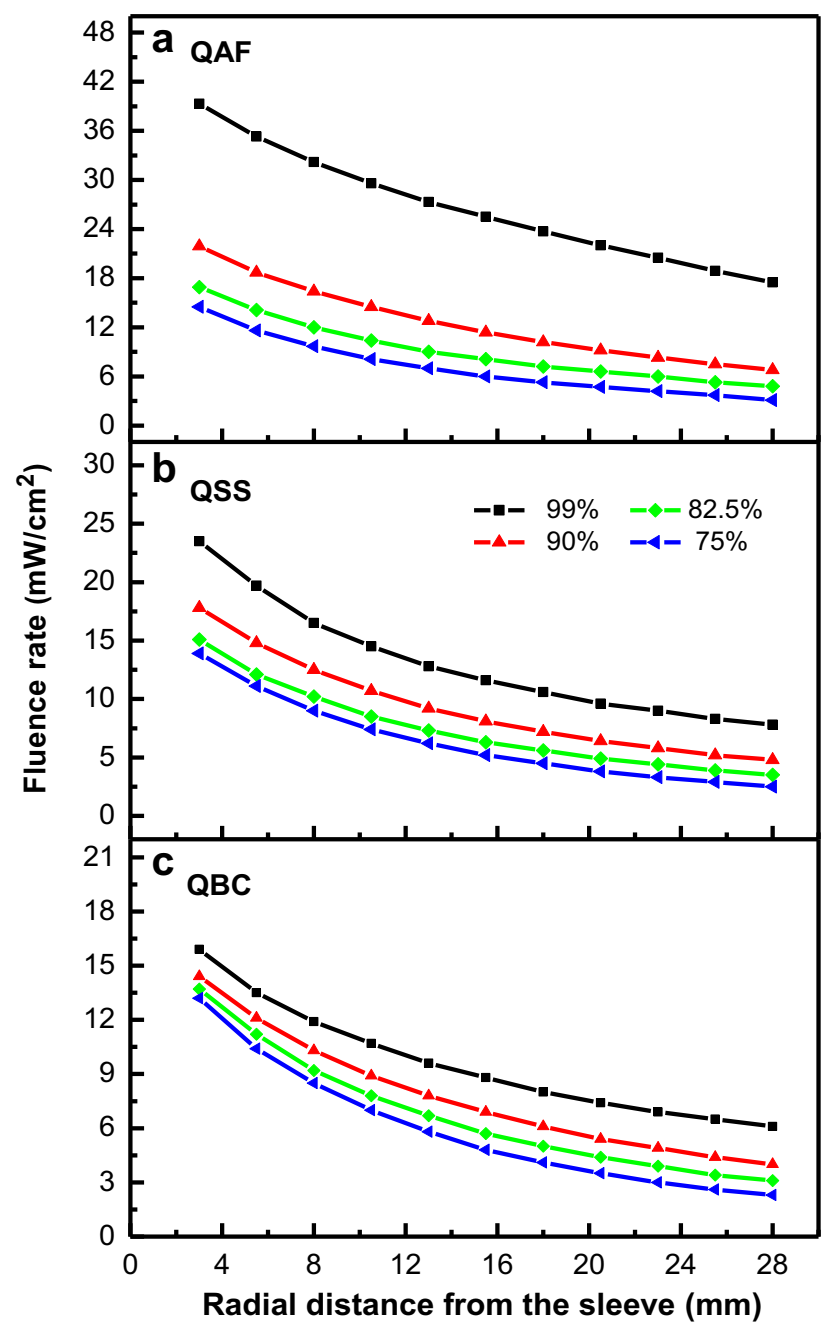

Fig. 5 - Fluence rate distribution in the central crosssection of the UV reactor with various inner-wall materials.

influence of reflective inner walls on the fluence can be evaluated approximately by the weighted average FRs and the ratios of walls QAF/QSS, QAF/QBC and QSS/QBC in the central cross-section of the UV reactor, as given in Table 1 (The method for calculating the weighted average FRs is given in Fig. S2.).

Comparing the QAF wall (reflection coefficient $=80.5 \%$ ) with the traditional QSS wall (reflection coefficient $=26.1 \%$ ), at a fixed UVT, the weighted average FR at each test point increased and the QAF/QSS wall ratio ranged from 1.08 to 2.09 (see Table 1). It is seen that the use of a highly reflective inner wall can significantly increase the fluence (or efficiency) of a UV reactor. The ratio of the weighted average FRs of QSS to QBC varied from 1.06 to 1.35 over the UVT range of $65-99 \%$. In fact, non-consideration of the impact of reflection from the inner wall is an important limitation of the current theoretical models. This study clearly shows that the presently used models can have an error of up to $35 \%$ as a result of the influence of reflection from the QSS inner wall. By combining with the traditional models, MFSD FR measurements can be used to evaluate the impact of the inner-wall reflection, such as the model error if neglecting the inner-wall reflection as 
Table 1 - Influence of various inner-wall materials on the weighted average FRs and their ratios in water with various UVTs.

\begin{tabular}{|c|c|c|c|c|c|c|}
\hline \multirow[t]{2}{*}{ UVT } & \multicolumn{3}{|c|}{$\mathrm{FR}_{\mathrm{wa}}\left(\mathrm{mW} / \mathrm{cm}^{2}\right)^{\mathrm{a}}$} & \multicolumn{3}{|c|}{$\mathrm{FR}_{\mathrm{wa}}$ ratio } \\
\hline & QAF & QSS & QBC & QAF/QSS & $\mathrm{QAF} / \mathrm{QBC}$ & QSS/QBC \\
\hline $99 \%$ & 24.60 & 11.75 & 8.73 & 2.09 & 2.82 & 1.35 \\
\hline $95 \%$ & 15.79 & 9.69 & 7.68 & 1.63 & 2.06 & 1.26 \\
\hline $92.5 \%$ & 13.74 & 8.55 & 7.12 & 1.61 & 1.93 & 1.20 \\
\hline $90 \%$ & 11.19 & 8.19 & 6.84 & 1.37 & 1.64 & 1.20 \\
\hline $87.5 \%$ & 10.38 & 7.67 & 6.34 & 1.35 & 1.64 & 1.21 \\
\hline $82.5 \%$ & 8.09 & 6.45 & 5.82 & 1.25 & 1.39 & 1.11 \\
\hline $75 \%$ & 6.13 & 5.37 & 4.99 & 1.14 & 1.23 & 1.08 \\
\hline $65 \%$ & 4.59 & 4.23 & 3.98 & 1.08 & 1.15 & 1.06 \\
\hline
\end{tabular}

well as the increase in weighted average FRs after adding a highly reflective inner wall.

\subsection{Reflection properties of various inner walls}

There are two kinds of reflection - specular and diffuse. Specular reflection is that obtained with a mirror in which the reflected image matches the illuminated object. On the other hand, in diffuse reflection, every element of the surface becomes a new UV source radiating in all directions outward from the surface. In the present system, if all the reflection is specular, therefore, the FR curves in Figs. 3 and 4 should have the same profile, except shifted upwards by the amount of reflection. In particular, the FR would be almost constant until near the end of the lamp. If the reflection is mostly diffuse, one should expect bell-shaped curves, as for the QAF case in Figs. 3 and 4 , since diffuse reflection would be highest near the central plane of the reactor.

The diffuse reflection properties of the three materials were measured at various incidence angles in this work (i.e., $20^{\circ}, 40^{\circ}$, and $60^{\circ}$ ). The results (Fig. 6) show that the reflection from all the three materials was neither absolute specular reflection nor absolute diffuse reflection. For ideal specular reflection, a slender reflection peak should be observed from the collimated incident beams. On the other hand, ideal diffuse reflection should not depend on the incidence angle, but have a cosine response to the acceptance angle. Fig. 6 also shows that the QAF curve is broader than the QSS and QBC curves at each incidence angle, implying that more UV beams impinging on the QAF wall were randomly scattered over a relatively wider range of acceptance angles. In fact, the stainless steel was mechanically polished with a glossy and smooth surface. In contrast, the aluminum foil had many wrinkles appearing on its surface. It is noted that the peak positions deviated from the corresponding incidence angles by about $5-13^{\circ}$. A possible reason is that the surfaces of the test materials could not keep ideally parallel to the sample plate in the vacuum UV analytical spectrophotometer.

Fig. 7 shows the distribution of experimental FR ratios as a function of the radial distance from the sleeve. It is evident that when the detector moved from the sleeve to the reactor wall, the FR ratios of walls QAF/QBC and QAF/QSS increased, and these increases were larger for the higher UVTs (Fig. 7a

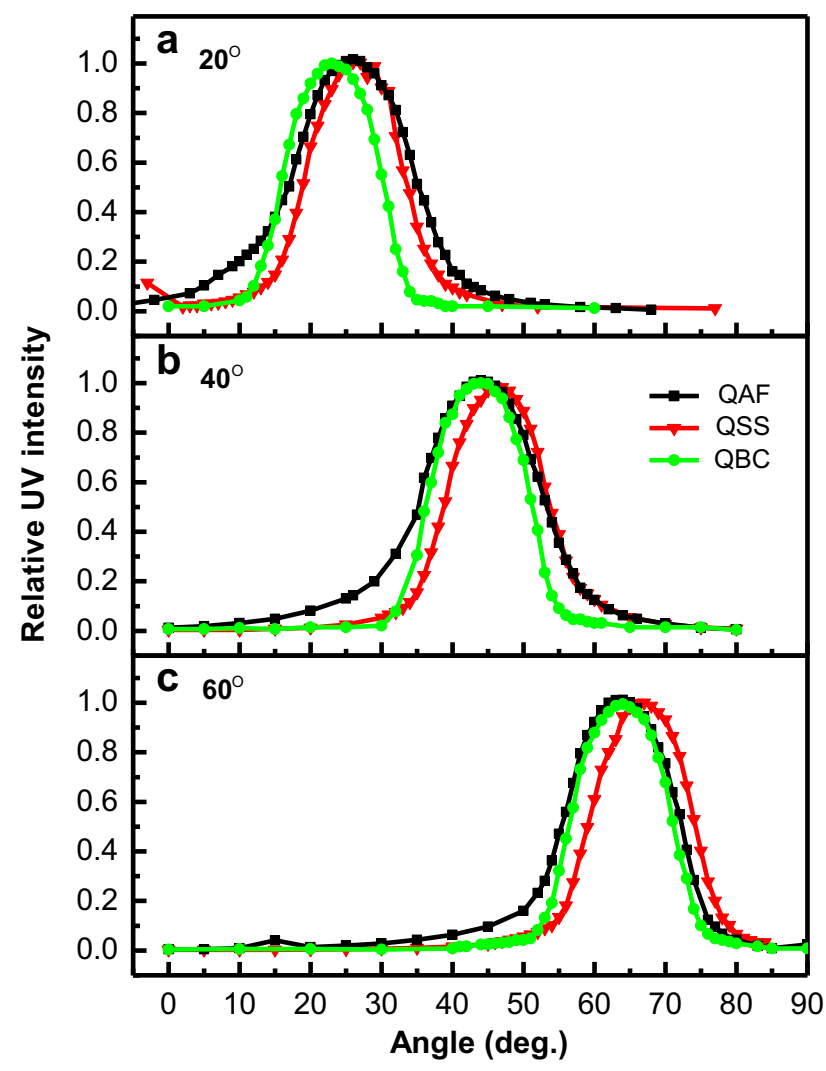

Fig. 6 - Diffuse reflection properties of various inner-wall materials tested at three incidence angles: (a) $20^{\circ}$, (b) $40^{\circ}$, and (c) $60^{\circ}$.

and c). In contrast, the FR ratio of walls QSS/QBC decreased at higher UVTs (90-99\%) while they increased at lower UVTs (75-82.5\%) (Fig. 7b). Because of the attenuation effect of the water, less reflected UV beams could reach the near-sleeve region. Hence in most cases, the FR ratio decreased toward the sleeve.

Note that the FR ratio of walls QSS/QBC decreased in water with high UVTs ( $\geq 90 \%$ ), while that of walls QAF/QBC increased when the detector was moved from the sleeve to the inner wall (Fig. 7a and b). One can conclude that the QAF case was more likely to increase the FR near the wall than for the QSS case in high UVT water. This arises from the different diffuse reflection properties of walls QAF and QSS. The QSS wall reflected the UV beams over a relative narrow range of angles (see Fig. 6), and more UV beams were reflected toward the center of the reactor than in the QAF case, which was able to scatter the UV over a wider range of angles. Likewise, near the sleeve, the test points of the QSS case could receive larger parts of total reflective UV beams from all inner-wall elements than in the QAF case. As a consequence, the QSS case led to a stronger reflection impact near the sleeve than the QAF case for high UVTs. However, with decreasing UVTs, the inner-wall reflection would enhance the FRs near the sleeve less significantly than those near the wall because of the longer path lengths of the reflected UV beams. Thus, the ratio of walls QSS/QBC increased as the test point moved toward the wall at UVTs $\leq 82.5 \%$, which is similar to those seen in Fig. 7a and c. 


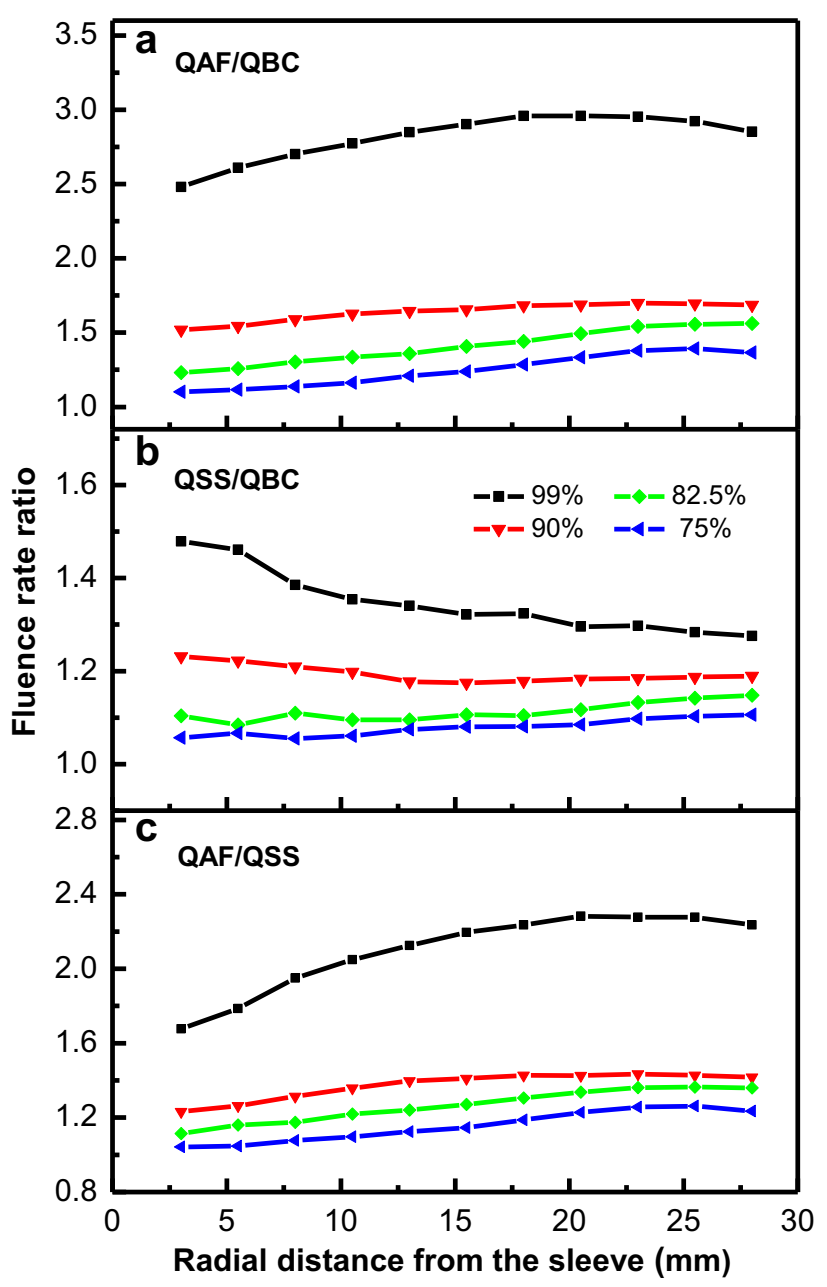

Fig. 7 - Fluence rate ratio distribution in the central crosssection of the UV reactor with various inner-wall materials: (a) QAF/QBC, (b) QSS/QBC, and (c) QAF/QSS.

This result shows that an inner wall, which can reflect the UV beams over a wider range of angles, could induce a more homogenous radial distribution of FR in the UV reactor.

\subsection{Importance for applications}

From the discussion above, the reflection coefficient and diffuse property of the inner wall are two important factors affecting the distribution of FR. In fact, the relatively lower FRs near the wall often reduce the disinfection efficiency of a UV reactor. This study shows that a high reflection coefficient and better diffuse property (i.e., scattering the UV beams to a wider range of angles) can increase the FR significantly and induce a more uniform FR distribution in the reactor, which leads to a notable increase in the reactor efficiency. The QAF wall had a higher reflection coefficient and a better diffuse property than the QSS wall, so the weighted average FRs increased accordingly. Polishing the inner wall has been adopted in most UV reactor manufacturing processes. However, this study suggests that the inner wall with a rough surface, which enhances more diffuse reflection, can produce a more uniform FR distribution. In addition, if there is a potential of fouling of the reflective wall, an anti-fouling material or a wall-cleaning apparatus should be considered when designing the UV reactor.

\section{Conclusions}

In this study, the FR distribution in a UV reactor with QAF, QSS, and QBC inner walls was determined in-situ by using a $360^{\circ}$ response MFSD. The tests were performed in water with various transmittances (UVTs) ranging from $65 \%$ to $99 \%$. Based on the experimental results, the following conclusions are drawn:

- A high reflection coefficient inner wall (e.g., QAF) can lead to a marked increase in the weighted average FRs, thus greatly improving the reactor efficiency.

- The FR distribution models, which neglect the influence of the inner-wall reflection, can have an error of up to $35 \%$ for the commonly used stainless-steel reactor.

- The uniformity of the FR distribution is strongly dependent on the diffuse reflection property of the inner wall, which leads to a better fluence delivery distribution in the UV reactor.

- This work helps to increase the accuracy of model predictions as well as optimize the design of high-efficiency UV reactors.

\section{Acknowledgments}

The work was financially supported by the National Natural Science Foundation of China (50921064, 20837001). Dr. Bolton thanks the Natural Sciences and Engineering Research Council of Canada (JRB) for its partial support of this study.

\section{Appendix A. Supplementary material}

Supplementary data related to this article can be found online at doi:10.1016/j.watres.2012.04.004.

\section{R E F E R E N C E S}

Blatchley III, E.R., 1997. Numerical modeling of UV intensity: application to collimated-beam reactors and continuous-flow systems. Water Research 31 (9), 2205-2218.

Blatchley III, E.R., Shen, C.Y., Scheible, O.K., Robinson, J.P., Ragheb, K., Bergstrom, D.E., Rokjer, D., 2008. Validation of large-scale, monochromatic UV disinfection systems for drinking water using dyed microspheres. Water Research 42 (3), 677-688.

Bohrerova, Z., Bohrer, G., Mohanraj, S.M., Ducoste, J., Linden, K.G., 2005. Experimental measurements of fluence distribution in a UV Reactor using fluorescent dyed microspheres. Environmental Science and Technology 39 (22), 8925-8930. 
Bolton, J.R., 2000. Calculation of ultraviolet fluence rate distributions in an annular reactor: significance of refraction and reflection. Water Research 34 (13), 3315-3324.

Bolton, J.R., 2010. Ultraviolet Applications Handbook, third ed. ICC Lifelong Learn Inc., Edmonton, $A B$, Canada.

Chen, J.Y., Deng, B.Q., Kim, C.N., 2011. Computational fluid dynamics (CFD) modeling of UV disinfection in a closedconduit reactor. Chemical Engineering Science 66 (21), 4983-4990.

Elyasi, S., Taghipour, F., 2010a. General method of simulating radiation fields using measured boundary values. Chemical Engineering Science 65 (20), 5573-5581.

Elyasi, S., Taghipour, F., 2010b. Simulation of UV photoreactor for degradation of chemical contaminants: model development and evaluation. Environmental Science and Technology 44 (6), 2056-2063.

Imoberdorf, G.E., Taghipour, F., Mohseni, M., 2008. Radiation field modeling of multi-lamp, homogeneous photoreactors. Journal of Photochemistry and Photobiology A-Chemistry 198 (2-3), 169-178.

Irazoqui, H.A., Cerdá, J., Cassano, A.E., 1973. Radiation profiles in an empty annular photoreactor with a source of finite spatial dimensions. AIChE Journal 19 (3), 460-467.

Irazoqui, H.A., Isla, M.A., Cassano, A.E., 2000. Simplified extense source model for photoreactor analysis and design. Industrial and Engineering Chemistry Research 39 (11), 4260-4271.

Jacob, S.M., Dranoff, J.S., 1970. Light intensity profiles in a perfectly mixed photoreactor. AIChE Journal 16 (3), 359-363.

Jin, S., Linden, K.G., Ducoste, J., Liu, D., 2005. Impact of lamp shadowing and reflection on the fluence rate distribution in a multiple low-pressure UV lamp array. Water Research 39 (12), 2711-2721.

Li, M.K., Qiang, Z.M., Li, T.G., Bolton, J.R., Liu, C.L., 2011. In situ measurement of UV fluence rate distribution by use of a micro fluorescent silica detector. Environmental Science and Technology 45 (7), 3034-3039.

Liu, D., Ducoste, J., Jin, S., Linden, K.G., 2004. Evaluation of fluence rate distribution models. Journal of Water Supply Research and Technology-Aqua 53 (6), 391-408.

Mamane-Gravetz, H., Linden, K.G., Cabaj, A., Sommer, R., 2005. Spectral sensitivity of Bacillus subtilis spores and MS2 coliphage for validation testing of ultraviolet reactors for water disinfection. Environmental Science and Technology 39 (20), 7845-7852.

Qualls, R.G., Johnson, J.D., 1983. Bioassay and dose measurement in UV disinfection. Applied and Environmental Microbiology 45 (3), 872-877.

Quan, Y., Ang, P.L., Ray, M.B., Pehkonen, S.O., 2005. Light distribution field in catalyst suspensions within an annular photoreactor. Chemical Engineering Science 60 (19), $5255-5268$.

Rahn, R.O., Bolton, J.R., Stefan, M.I., 2006. The iodide/iodate actinometer in UV disinfection: determination of the fluence rate distribution in UV reactors. Photochemistry and Photobiology 82 (2), 611-615.

Sommer, R., Cabaj, A., Haider, T., 1996. Microbicidal effect of reflected UV radiation in devices for water disinfection. Water Science and Technology 34 (7-8), 173-177.

Sozzi, D.A., Taghipour, F., 2006. UV reactor performance modeling by Eulerian and Lagrangian methods. Environmental Science and Technology 40 (6), 1609-1615. 